\title{
Zig-zag networks of self-excited periodic oscillations in a tunnel diode and a fiber-ring laser
}

\author{
Ricardo E. Francke, ${ }^{1}$ Thorsten Pöschel,,${ }^{2,3}$ and Jason A. C. Gallas ${ }^{1,2,3,4}$ \\ ${ }^{1}$ Instituto de Física, Universidade Federal do Rio Grande do Sul, 91501-970 Porto Alegre, Brazil \\ ${ }^{2}$ Institute for Multiscale Simulations, Friedrich-Alexander-Universität Erlangen-Nürnberg, 91052 Erlangen, Germany \\ ${ }^{3}$ Departamento de Física, Universidade Federal da Paraíba, 58051-970 João Pessoa, Brazil \\ ${ }^{4}$ Instituto de Altos Estudos da Paraíba, Rua Infante Dom Henrique 100-1801, 58039-150 João Pessoa, Brazil
}

(Received 30 January 2013; published 9 April 2013)

\begin{abstract}
We report numerical evidence showing that periodic oscillations can produce unexpected and wide-ranging zigzag parameter networks embedded in chaos in the control space of nonlinear systems. Such networks interconnect shrimplike windows of stable oscillations and are illustrated here for a tunnel diode, for an erbium-doped fiber-ring laser, and for the Hénon map, a proxy of certain $\mathrm{CO}_{2}$ lasers. Networks in maps can be studied without the need for solving differential equations. Tuning parameters along zig-zag networks allows one to continuously modify wave patterns without changing their chaotic or periodic nature. In addition, we report convenient parameter ranges where such networks can be detected experimentally.
\end{abstract}

DOI: 10.1103/PhysRevE.87.042907

PACS number(s): 05.45.Ac, 05.45.Pq, 89.75.Kd

\section{INTRODUCTION}

The complex behavior of simple dissipative systems with a small number of degrees of freedom has been intensively studied in a variety of fields. For many decades, the great interest has been to investigate mainly the structure of the phase space of flows, with particular emphasis on the possible transitions from order to chaos and a plethora of instabilities associated with these transitions [1-3]. More recently, extensive numerical simulations have revealed unexpected regularities in a complementary setting, namely, in the control parameter space of systems as diverse as electronic circuits, laser systems, and modulational interactions in a plasma, in chemical and biophysical oscillators, and in many other paradigmatic flows covering a large spectrum of practical applications [4-21]. Such regularities emerged while attempting to classify systematically all collective oscillations supported by the aforementioned applications.

As an example of a wide-ranging regularity in parameter space we mention the infinite alternation of spirals of chaos and of periodicity which emanate from certain periodicity $h u b s$, namely, from exceptional focal points ubiquitous in the control space of some systems [8]. In this alternation, each spiral corresponds to a certain periodic phase characterized individually by an infinite cascade of periodic waveforms specific to it which evolve and get continuously more complex when parameters are suitably changed along the spiral towards its focus. Their period seems to accumulate to a specific value near the focal point while the number of peaks (local maxima) in each period seems to grow without bound, diverging as one approaches the focal point more and more [8,9]. Knowledge of such infinite networks of periodic phases has a direct practical application. Since changes between spirals correspond to changes between distinct families of oscillatory wave patterns, spiral networks provide a unique possibility for selectively switching from one family of patterns to another.

The aim of this paper is to report numerical evidence showing that regular oscillations in nonlinear systems can emerge, forming a novel kind of wide-ranging network, namely, certain zig-zag networks of periodic phases embedded in chaos. The nodes composing such networks are "shrimps" [22-26], i.e.,

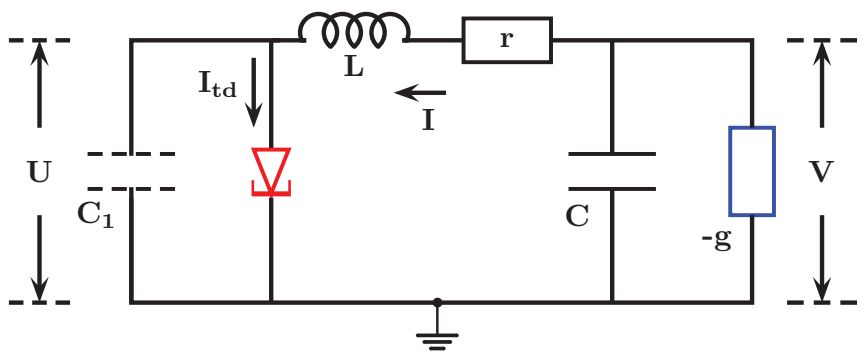

FIG. 1. (Color online) Schematic representation of the tunnel diode circuit where zig-zag networks were detected. This circuit leads to the dissipative flow of Eqs. (5)-(7). The voltage applied to the diode is denoted by $U$. Dashed lines are used to represent the parasitic capacitance $C_{1}$.

the complex mosaic formed by periodic and chaotic windows shown below in Fig. 5(d), while the edges are shrimp legs. We describe zig-zag networks explicitly for a tunnel diode, a solid-state device, for an erbium-doped fiber-ring laser, and for the Hénon map, a system that describes well certain driven $\mathrm{CO}_{2}$ lasers $[10,27,28]$. In this latter example, the network interconnects hitherto unexplored islands characterized by relatively high periodicities and has the significant advantage of providing a framework for studying networks without the need for solving differential equations. For each example, we specify convenient parameter windows that should be helpful in locating networks experimentally. We start by describing networks found in the electronic circuit with a tunnel diode. We use the known configuration (Fig. 1) considered in pioneering works by Pikovsky and Rabinovich [29-31].

Before proceeding, recall that so far there are no practical mathematical tools capable of predicting hubs and spirals, their location, their structural complexities, their phase boundaries, and the unfolding of their intricate waveforms and their bifurcations. Of course, there are several powerful mathematical techniques, like continuation methods and the study of global homoclinic bifurcations, that give some insights for some aspects of these structures in parameter space [4-6]. However, note that parameter space knowledge about hubs, 
about periodic phases with arbitrarily high periods, and about chaotic phases needs to be extracted in an independent way, via numerical simulations of the corresponding flows, particularly in new scenarios dealing with interesting and unexplored phenomena that are known not to be at all associated with homoclinic bifurcations [7].

\section{THE FLOW OF THE TUNNEL DIODE CIRCUIT}

According to Kirchhoff's laws, the flow defined by the self-excited (autonomous) oscillator with the tunnel diode is

$$
\begin{aligned}
& V-U=r I+L \frac{d I}{d t}, \\
& -I=-g V+C \frac{d V}{d t}, \\
& I=F(U)+C_{1} \frac{d U}{d t},
\end{aligned}
$$

where $I$ denotes the current through the inductance, $U$ is the voltage across $C_{1}$, and $V$ is the voltage across $C$. With the help of the voltage $W \equiv V-r I$ between $L$ and $r$, introducing the convenient change of variables $I=(x+1) I_{0}, U=(z+1) U_{0}$, $y=\left(W-U_{0}\right) /\left(\omega L I_{0}\right)$, and $\tau=\omega t$, where

$$
\omega \equiv \sqrt{\frac{1-g r}{L C}},
$$

and replacing $F(U)$ by $f(z)$, one finds the following handy adimensional equations for the flow generated by the diode:

$$
\begin{gathered}
\frac{d x}{d \tau}=y-\delta z, \\
\frac{d y}{d \tau}=-x+2 \gamma y+\alpha z+\beta, \\
\mu \frac{d z}{d \tau}=x-f(z),
\end{gathered}
$$

where the five parameters denote the following combinations of reactances and conditions:

$$
\begin{gathered}
\delta=\frac{U_{0}}{\omega I_{0} L}, \quad 2 \gamma=\frac{g L-r C}{\omega L C}, \quad \alpha=\frac{r U_{0}}{\omega^{2} L^{2} I_{0}}, \\
\beta=-1+\frac{g U_{0}}{\omega^{2} I_{0} L C}=\alpha-1+2 \gamma \delta, \quad \mu=\frac{\omega C_{1} U_{0}}{I_{0}} .
\end{gathered}
$$

These expressions show that specific values of $\delta, \gamma, \alpha, \beta$, and $\mu$ may be conveniently reached in several different ways by suitably combining the reactances and conditions involved. This clearly shows that all circuit elements are equally important for the dynamics, not just the tunnel diode, the nonlinear element.

Equations (5)-(7) coincide with the equations investigated by Pikovsky and Rabinovich [29-31]. However, our equations contain $\omega^{2}=(1-g r) /(L C)$ instead of the approximate result $\omega^{2}=1 /(L C)$. Both sets of equations agree in the limit $g r \ll 1$. For simplicity, following Pikovsky and Rabinovich [29-31], we assume the characteristic function of the tunnel diode in Eq. (7) to be a cubic function, namely, $f(z) \equiv z^{3}-z$.

The flow of the tunnel diode was investigated previously by Carcasses and Mira [32]. However, these authors used a Poincaré surface of section to associate a two-dimensional diffeomorphism $T$ to the differential equations and studied qualitatively bifurcations not of the flow but of the mapping $T$ as seen in the $\mu \times \beta$ parameter plane. Here, we consider the bifurcation structure of the flow itself, Eqs. (5)-(7), not of an approximate Poincaré proxy.

\section{ZIG-ZAG NETWORKS IN THE TUNNEL DIODE}

Figure 2 shows three high-resolution stability diagrams classifying the dynamical behaviors present in the diode circuit. A glance at Fig. 2 is enough to convince one that periodic and chaotic oscillations are sprinkled in a rather complex way in the $\gamma \times \delta$ control parameter plane. First, one realizes that the control plane is subdivided macroscopically

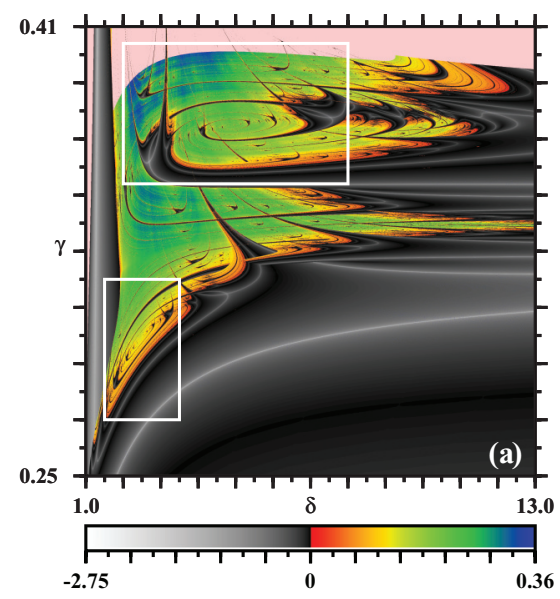

(a) Global view

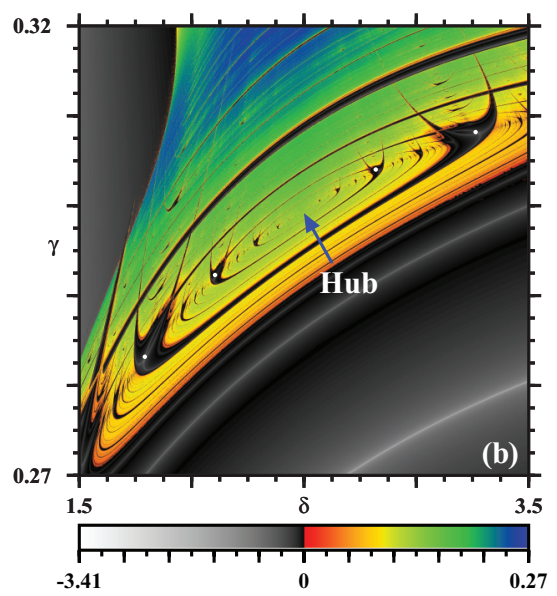

(b) anti-clockwise spiral

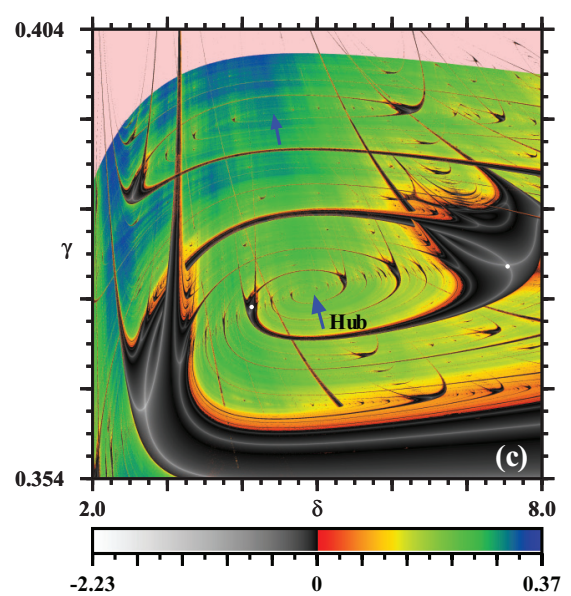

(c) clockwise spiral

FIG. 2. (Color online) Lyapunov phase diagrams for the tunnel diode circuit, Eqs. (5)-(7). (a) Global view of the control space. The right corner of the upper box contains several zig-zag networks, shown magnified in Fig. 3. (b) Enlargement of the lower box in (a), illustrating a large anticlockwise spiraling network emanating from the focal hub indicated by the arrow. (c) Enlargement of the upper box in (a) showing clockwise spiraling networks, two of them with hubs indicated by arrows. The darker (pink) stripe on the top of (a) and (c) marks divergent solutions. Here $\alpha=-0.013, \beta=0, \mu=1.0$. Each individual panel displays $2400 \times 2400=5.76 \times 10^{6}$ Lyapunov exponents. 
into two distinct phases, periodic and chaotic, which have rather complicated shapes and boundaries. Before describing the diagrams individually, we first say a few words about how they were computed.

Our stability diagrams were obtained by solving numerically Eqs. (5)-(7) with a standard fixed-step fourth-order Runge-Kutta integrator and using the solutions obtained to compute all three Lyapunov exponents of the system according to the method of Wolf et al., as described in Ref. [23], and plotting the largest nonzero exponent, following a standard procedure described in detail elsewhere [9]. For each parameter point on a $2400 \times 2400$ grid of points we scanned the phase space, recording all stable asymptotic oscillations. Such classification is a rather demanding computational task that was performed with the help of a SGI Altix cluster made of 1536 AMD Opteron processors running at $2.3 \mathrm{GHz}$ together with software that we developed in house specifically for this purpose. As is well known [1], Lyapunov exponents are useful indicators capable of discriminating the nature of the asymptotic oscillations, i.e., discriminating between periodic oscillations (characterized by negative exponents) and chaos (positive exponents). As usual for nonlinear flows, the diode displays multistability, i.e., for a given set of parameters it is possible to find several distinct stable periodic and/or chaotic oscillations, depending on the initial conditions [1]. In such cases, we selected the color to be plotted so as to maximize the phase of periodic oscillations and the phenomena of main interest to us, as discussed below.

Figure 2(a) shows a large portion of the diode control space. It contains two boxes which are enlarged in Figs. 2(b) and 2(c). These enlargements serve to show where the zig-zag networks are located and to illustrate a nice by-product of the diode circuit: its control space displays several large groups of nested spiral networks and hubs of the type mentioned in the Introduction, not just single spirals as known so far [8,9]. The discovery of a circuit with several large spirals is important because such a circuit can help bypass the major obstruction in the experimental and theoretical study of spirals: the fact that known spirals are strongly distorted and compressed due to the scales used. With hindsight, after spirals are duly located, it is, of course, possible to search for a rescaling of the flow in order to produce nice looking spirals. But this is a second stage in the study. First, one needs to find the spirals, something that can be quite difficult (not to say impossible) without the help of extensive numerical calculations. Of course, distortions are associated with the fact that, so far, all known differential equations displaying spirals represent physical phenomena; i.e., they represent real-life physical flows rather than mathematical "normal forms" suitably constructed in an ad hoc manner for pure mathematical investigations. Clearly, it would be very useful to find means of divining flows with large groups of wide spirals and hubs, particularly if based on real-life easily accessible physical coordinates, to facilitate both experimental and theoretical or numerical studies.

The parameter region shown in Fig. 3(a) contains a plethora of zig-zag networks interconnecting families of periodic oscillations. The largest such network connects the wide shrimp clusters labeled $P, Q, R$, and $S$. Another zig-zag network exists inside the white box, shown magnified in Fig. 3(b), where its first few alternations are labeled $A, B$, and $C$. The basic signature of zig-zag patterns is the alternation of three shrimps, exemplified by the sequences $P Q R$ and $Q R S$ in Fig. 3(a) and $A B C$ in Fig. 3(b). For brevity, we call such triplets "V connections" or "V bridges." These connections are present in Fig. 2 of a recent paper by Manchein et al. [33], who used the discrete map of Carlo et al. [34] to study the distribution of unbiased current in the ratchet transport of particles [35].

The shrimp-shaped windows of the $\mathrm{V}$ bridges are the same ones that twist continuously either clockwise or counterclockwise, as shown in Fig. 2, to form the spiral networks discussed in the Introduction. Originally [22-24,26], such shrimps were found to form infinite sequences of essentially

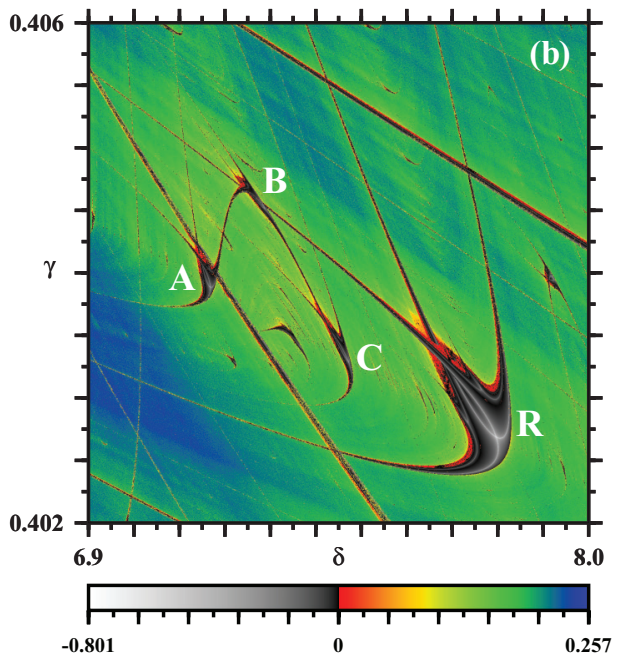

FIG. 3. (Color online) Examples of V connections in the control space of the tunnel diode. (a) A zig-zag pattern $P Q R S$ formed by "gluing" the $\mathrm{V}$ connections together. The zig-zag continues beyond $S$, but the additional alternations are too small to be seen in this scale. The upper dark (pink) background denotes unbounded solutions (divergence) and is riddled with shrimps, e.g., $Q$ and $S$. (b) Magnification of the V connection $A B C$ in the white box in (a). One of the legs of $R$ allows passing between $R$ and $B$ via continuous parameter changes. Here $\alpha=-0.33, \beta=0, \mu=1.0$. 
parallel structures, apparently completely disconnected from each other. Subsequently, 15 years later, these windows were discovered to emerge interconnected, forming continuous spiral networks [8]. In Fig. 3(b), however, shrimps $A$ and $C$ are clearly interconnected with $B$, forming a structure that resembles an upside-down V. From additional magnifications of specific regions of Fig. 3 (not shown) it is possible to recognize the unfolding and interconnection of several $\mathrm{V}$ bridges that form a long chain, a zig-zag network. In such networks, however, each successive $\mathrm{V}$ bridge gets strongly compressed, making it difficult to assert whether the zig-zag network is infinite or not.

\section{BRIDGES IN A LASER AND IN THE HÉNON MAP}

The purpose of this section is to show that the same connections described above exist also in the flow governing an erbium-doped dual-ring fiber laser and in a celebrated textbook example of the discrete-time model, the Hénon map. We start by computing stability diagrams for an erbium-doped dual-ring fiber laser with the lasing fields in the two rings frequency locked through a coupler $c_{0}$ with a phase change of $\pi / 2$ from one ring to the other. In this configuration, the laser equations for the fundamental system are [3,36-38]

$$
\begin{gathered}
\frac{d E_{a}}{d t}=-\left(E_{a}+c_{0} E_{b}\right) k_{a}+g_{a} E_{a} D_{a}, \\
\frac{d E_{b}}{d t}=-\left(E_{b}-c_{0} E_{a}\right) k_{b}+g_{b} E_{b} D_{b}, \\
\frac{d D_{a}}{d t}=-\left(1+I_{p a}+E_{a}^{2}\right) D_{a}+I_{p a}-1, \\
\frac{d D_{b}}{d t}=-\left(1+I_{p b}+E_{b}^{2}\right) D_{b}+I_{p b}-1,
\end{gathered}
$$

where $E_{a}$ and $E_{b}$ are the lasing fields and $D_{a}$ and $D_{b}$ are the population inversions in rings $a$ and $b$, respectively. The parameters $k_{a}, k_{b}, g_{a}$, and $g_{b}$ represent the decay rate and the gain coefficient of lasing fields $a$ and $b$, as indicated. $I_{p a}$ and $I_{p b}$ represent pump intensity in the respective fiber rings. Note that this laser model contains cubic nonlinearities, similar to the nonlinearity present in the tunnel diode flow. As for parameter values, in an interesting paper, Zhang and Shen [38] detected hyperchaotic dynamics for the following set of parameters: $k_{a}=k_{b}=1000, c_{0}=0.2, g_{a}=10500, g_{b}=$ 4700, parameters that for simplicity we adopt here. For them we computed stability diagrams as a function of the pump intensities $I_{p a}$ and $I_{p b}$.

Figure 4 illustrates a typical Lyapunov stability diagram obtained for the laser where, as before, periodic and chaotic laser oscillatory phases are discriminated by the colors of their exponents. Inside box $\mathrm{A}$ in this figure it is possible to recognize the characteristic $\mathrm{V}$ bridge discussed above. Several other interconnections like this one exist in the system. We also computed several additional diagrams (not shown) for a number of parameter combinations "centered" around the above ones. Such simulations showed that the laser phases are robust against parameter fluctuations and that Fig. 4 is representative of the distribution of periodic and chaotic oscillations in the laser. The main effect of changing the above parameters is to produce small shifts of the structures seen

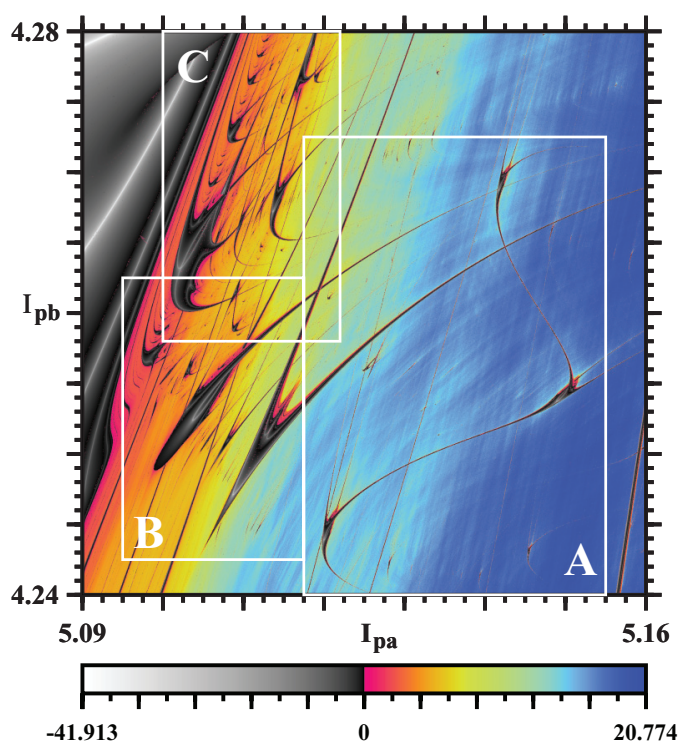

FIG. 4. (Color online) Lyapunov stability diagram showing V connections for an erbium-doped fiber-ring laser (inside box A). After shrimps, the cuspidal and rounded periodicity regions seen in box $\mathrm{B}$ are the most frequently observed shapes of periodicity windows. Box $\mathrm{C}$ is rich in complicated structures that are difficult to classify systematically. This diagram displays $2400 \times 2400=5.76 \times 10^{6}$ parameter points.

in the stability diagram as well as changes of their relative areas [3].

As a curious byproduct, note the region delimited by box $\mathrm{B}$ in Fig. 4. In its lower right corner one finds a laser phase with the shape of a sharp cusp and, to its left, a large rounded phase. This pair of structures appears profusely in the stability diagram. In fact, after the ubiquitous shrimps, these rounded and cuspidal phases are the structures most frequently observed in flows and maps. Their detailed structure has not been studied completely so far, although some results are available [39]. Box $\mathrm{C}$ contains a huge number of interesting periodicity phases with rather complex structures. They obviously deserve to be better investigated.

Is it possible to find $\mathrm{V}$ bridges in simple discrete-time systems, i.e., in mappings? This possibility is useful because it would allow the investigation of parameter networks without the need for solving differential equations, thereby avoiding the familiar errors associated with numerical integrations. As already mentioned, the ratchet map of Carlo et al. contains $\mathrm{V}$ bridges [33,34]. With no difficulty, we found several $\mathrm{V}$ connections in the very first map where we looked for them, namely, in the Hénon map, the paradigmatic multidimensional dissipative system [22-26],

$$
(x, y) \mapsto\left(a-x^{2}+b y, x\right) \equiv H_{a, b}(x, y) .
$$

As in Ref. [22], Fig. 5(a) shows a stability diagram of the most relevant portion of its control parameter space. For each individual $k$-periodic orbit we also determined the trace $\tau$ of the Jacobian of $H_{a, b}^{k}$, the $k$ th composition of the map. Then, instead of using a solid color to paint the whole periodic phase, we partitioned it into two sectors. When $\tau>0$, we represented the period using the colors in the color bar, using black to paint the periodic portion where $\tau<0$. This artifact increased the 

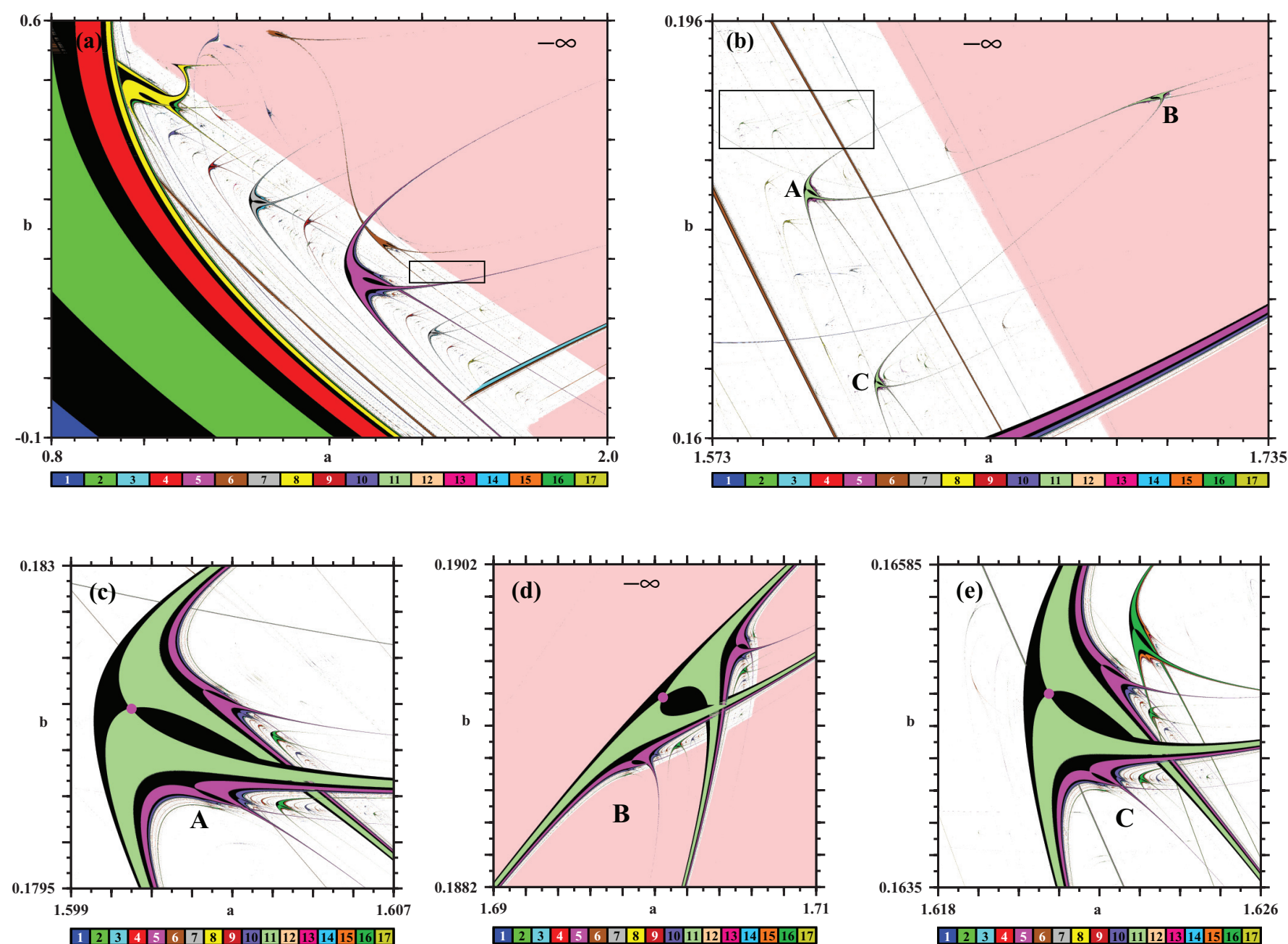

FIG. 5. (Color online) Illustration of V connections in the Hénon map. (a) Global view showing shrimps and complex structures embedded in the chaotic white background. The darker (pink) background denotes divergence. The periods are as indicated in the color bar. (b) Magnification of the box in (a) showing a $\mathrm{V}$ connection formed by three period-11 shrimps labeled $A, B, C$. The box contains additional period-22 V connections which are too small to be visible at this scale. Their "heads" [22,23] are given in Table I. The three panels in the bottom row display magnifications of shrimps (c) $A$, (d) $B$, and (e) $C$ with their heads marked by magenta dots, defined in Table I. (d) shows a shrimp in its pure state, i.e., in a state where it is trivial to see where its chaotic phase ends, something not recognizable in (c) or (e) (see text). Each panel displays the analysis of $2400 \times 2400=5.76 \times 10^{6}$ parameter points.

information content of the diagrams by displaying the inner structure of each periodicity cell, analogous to plotting the "multiplier" of two-parameter one-dimensional maps [23]. As seen from Figs. 5(c)-5(e), the "center" of each periodicity window then becomes visible (compare with Fig. 3 of Ref. [22] or Fig. 2(b) of Ref. [26] where centers cannot be seen).

A few $\mathrm{V}$ bridges of the Hénon map occur inside the black box in Fig. 5(a), magnified in Fig. 5(b). In this figure, the most easily visible bridge is formed by shrimps $A, B$, and $C$, shown in detail in Fig. 5(c)-5(e). Noteworthy in Fig. 5(b) is the boundary between the white background, representing chaos, and the darker (pink) background, representing unbounded oscillations (divergence). This background of divergence can be conveniently used to display shrimps in their "pure" state: in Fig. 5(d) it is trivial to see where the chaotic phase of shrimp B ends. while in Figs. 5(c) and 5(e) this transition cannot be identified because of the continuous transition to the regular chaotic phase present in the background. Moving out from shrimp $B$ has catastrophic consequences, with the system losing stability and moving to the attractor located at infinity (divergence), instead of starting to oscillate in a new chaotic trajectory, which happens when moving out from either shrimp $A$ or $C$.

In addition to the $\mathrm{V}$ bridges described, the Hénon map contains a large number of other connections with rather complex forms that are difficult to classify and that are still awaiting a systematic study. The paper of Lorenz [26] may be regarded as a step in this direction. In the Hénon map, the number of complex interconnections is so great that one has the impression that, in the end, all periodicity clusters might in fact compose just a huge single network of interconnected domains. This means that by suitably selecting parameters one could navigate around the whole network, moving from one periodic oscillation to another, without ever needing to cross the large sea of chaos densely surrounding the network everywhere. This is a remarkable and potentially 
TABLE I. Coordinates $(a, b)$ and one orbital point $(x, y)$ near the "head" of the period-11 shrimps, indicated by magenta dots in Figs. 5(c)-5(e), forming the $\mathrm{V}$ bridges labeled $A, B$, and $C$. The four additional points are from $\mathrm{V}$ connections inside the box in Fig. 5(b).

\begin{tabular}{lllcc}
\hline \hline Period & \multicolumn{1}{c}{$a$} & \multicolumn{1}{c}{$b$} & \multicolumn{1}{c}{$x$} & $y$ \\
\hline 11 & 1.6005 & 0.18145 & 1.3952093 & 0.0029855 \\
11 & 1.7005 & 0.189375 & 1.4800079 & 0.0056994 \\
11 & 1.62075 & 0.16491 & 1.4307282 & 0.0040349 \\
22 & 1.58038 & 0.1866968 & 1.3713907 & 0.0018595 \\
22 & 1.5950167 & 0.1874798 & 1.3841817 & 0.0024175 \\
22 & 1.581413 & 0.1861185 & 1.3729099 & 0.0019164 \\
22 & 1.5863495 & 0.1868117 & 1.3768124 & 0.0021038 \\
\hline \hline
\end{tabular}

very useful property of parameter networks of any possible shape.

One may wonder how complex connections and wide parameter networks could have remained unnoticed despite the ever-flowing deluge of papers devoted to multidimensional maps. A plausible explanation lies in the relatively high periods of the orbits associated with $\mathrm{V}$ bridges, a regime that still remains largely unexplored. A detailed investigation of $\mathrm{V}$ bridges in the Hénon map together with their metric properties and scalings is presented elsewhere [19]. While it seems likely that global bifurcations lurk behind the $\mathrm{V}$ bridges since they appear in systems having rather distinct properties, it seems too premature to try to explain their origin before performing a thorough investigation of these systems.

\section{CONCLUSIONS AND OUTLOOK}

We found that tunnel diodes produce unexpected and rich dynamical behaviors such as $\mathrm{V}$ bridges and zig-zag networks. The circuit with a tunnel diode is particularly well adapted for experimental investigations not only of the zig-zag networks but also of the more familiar spiral networks because it contains surprisingly large and relatively undistorted spirals (Fig. 2). Zig-zag networks seem to be rather ubiquitous since they can be found with no special effort in other systems like the erbium-doped dual-ring fiber laser and the Hénon map. Figure 2 provides an example of an unexpected system displaying spirals that twist continuously both clockwise and counterclockwise in control space.
Knowledge of parameter networks allows one to effectively control the dynamics in a predictable and stable (permanent) way. Recall that popular control techniques rely on the application of series of infinitesimal parameter changes which are unable to target preassigned and stable behaviors [1]. Such procedures lead to random final destinations and require the permanent application of "perturbations" to the system in order to maintain operation of the unstable random destination. In sharp contrast, parameter networks can be used (i) as guides to implement parameter changes of any arbitrary size, (ii) to move to any preassigned state, and (iii) to perform changes only among stable oscillations, either with a single parameter jump or, if needed or desired, using sequences of controlled parameter changes.

We hope this work will trigger research about parameter networks both experimentally and theoretically. Possible theoretical directions involve developing tools to anticipate where networks might be located in stability diagrams and, more importantly, which type of nonlinearities might produce them. Also of interest is to investigate what happens with the symbolic coding of orbits belonging to $\mathrm{V}$ connections (which contain several cusps) when following closed paths around them. It is known that in some regions of control space, circulations around closed parameter paths involve unavoidable ambiguities in the symbolic coding [25]. At present, we are still waiting for the development of a mathematical framework capable of addressing these types of problems. The origin and structural properties of parameter networks in dynamical systems are far from being understood except for a growing number of explicit examples. Zig-zag networks made of $\mathrm{V}$ bridges provide a new and potentially fruitful addition to the arsenal of networks allowing one to prospect the global organization of stable oscillations so vital for applications.

\section{ACKNOWLEDGMENTS}

We are indebted to Arkady Pikovsky for helpful exchanges concerning certain approximations used in the pioneering work of Refs. [29-31]. This work was supported by the Deutsche Forschungsgemeinschaft through the Cluster of Excellence "Engineering of Advanced Materials." J.A.C.G. was also supported by CNPq, Brazil.
[1] Y. C. Lai and T. Tél, Transient Chaos (Springer, New York, 2011).

[2] D. M. Kane and K. A. Shore, Unlocking Dynamical Diversity (Wiley, New York, 2005).

[3] R. E. Francke, T. Pöschel, and J. A. C. Gallas, in Advances in Nonlinear Dynamics Synchronization with Selected Applications in Theoretical Electrical Engineering, edited by K. Kyamakya, W. A. Halang, W. Mathis, J. C. Chedjou, and Z. Li (Springer, Berlin, 2013), Chap. 9.

[4] R. Vitolo, P. Glendinning, and J. A. C. Gallas, Phys. Rev. E 84, 016216 (2011)

[5] R. Barrio, F. Blesa, S. Serrano, and A. Shilnikov, Phys. Rev. E 84, 035201(R) (2011).
[6] R. Barrio, F. Blesa, and S. Serrano, Phys. Rev. Lett. 108, 214102 (2012).

[7] J. G. Freire and J. A. C. Gallas, Phys. Rev. E 82, 037202 (2010).

[8] C. Bonatto and J. A. C. Gallas, Phys. Rev. Lett. 101, 054101 (2008); Philos. Trans. R. Soc. A 366, 505 (2008); C. Bonatto, J. A. C. Gallas, and Y. Ueda, Phys. Rev. E 77, 026217 (2008).

[9] For a survey see J. A. C. Gallas, Int. J. Bifurcation Chaos Appl. Sci. Eng. 20, 197 (2010), and references therein.

[10] C. Bonatto, J. C. Garreau, and J. A. C. Gallas, Phys. Rev. Lett. 95, 143905 (2005).

[11] V. Kovanis, A. Gavrielides, and J. A. C. Gallas, Eur. Phys. J. D 58, 181 (2010). 
[12] J. G. Freire T. Pöschel, and J. A. C. Gallas, Europhys. Lett. 100, 48002 (2012); J. G. Freire and J. A. C. Gallas, Phys. Chem. Chem. Phys. 13, 12191 (2011); Phys. Lett. A 375, 1097 (2011).

[13] D. Laroze et al., IEEE Trans. Magn. 48, 3567 (2012); J. Bragard, H. Pleiner, O. J. Suarez, P. Vargas, J. A. C. Gallas, and D. Laroze, Phys. Rev. E 84, 037202 (2011).

[14] L. Junges and J. A. C. Gallas, Phys. Lett. A 376, 2109 (2012); Opt. Commun. 285, 4500 (2012).

[15] D. F. M. Oliveira, M. Robnik, and E. D. Leonel, Chaos 21, 043122 (2011)

[16] D. F. M. Oliveira and E. D. Leonel, Phys. Lett. A 376, 3630 (2012); New J. Phys. 13, 123012 (2011).

[17] C. Stegemann, H. A. Albuquerque, R. M. Rubinger, and P. C. Rech, Chaos 21, 033105 (2011).

[18] E. V. Viana, R. M. Rubinger, H. A. Albuquerque, A. G. de Oliveira, and G. M. Ribeiro, Chaos 20, 023110 (2010).

[19] G. M. Ramírez-Ávila, T. Pöschel, and J. A. C. Gallas (unpublished).

[20] R. A. Miranda, E. L. Rempel, and A. C. L. Chang, Phys. Plasmas 19, 112303 (2012), and references therein.

[21] E. S. Medeiros, R. O. Medrano, I. L. Caldas, and S. L. T. de Souza, Phys. Lett. A 377, 628 (2013).

[22] J. A. C. Gallas, Phys. Rev. Lett. 70, 2714 (1993).

[23] J. A. C. Gallas, Phys. A 202, 196 (1994); Appl. Phys. B 60, S-203 (1995); A. R. Zeni and J. A. C. Gallas, Phys. D 89, 71 (1995).

[24] B. R. Hunt, J. A. C. Gallas, C. Grebogi, J. A. Yorke, and H. Koçak, Phys. D 129, 35 (1999).
[25] A. Endler and J. A. C. Gallas, Phys. Rev. E 65, 036231 (2002).

[26] E. N. Lorenz, Phys. D 237, 1689 (2008).

[27] C. L. Pando, G. A. Luna Acosta, R. Meucci, and M. Ciofini, Phys. Lett. A 199, 191 (1995).

[28] E. J. Doedel and C. L. Pando L., Phys. Rev. E 84, 056207 (2011); Int. J. Bifurcation Chaos Appl. Sci. Eng. 22, 1250238 (2012).

[29] A. S. Pikovsky and M. Rabinovich, Sov. Phys. Dokl. 23, 183 (1978); Dokl. Akad. Nauk SSSR 239, 301 (1978).

[30] M. Rabinovich, Sov. Phys. Usp. 21, 443 (1978); Usp. Fiz. Nauk 125, 123 (1978).

[31] A. S. Pikovsky and M. Rabinovich, Phys. D 2, 8 (1981).

[32] J. P. Carcasses and C. Mira, in Proceedings of the International Conference on Iteration Theory: ECIT 89, Batschuns, edited by C. Mira, N. Netzer, C. Simo, and G. Targonski (World Scientific, Singapore, 1991), pp. 29-41.

[33] C. Manchein, A. Celestino, and M. W. Beims, Phys. Rev. Lett. 110, 114102 (2013).

[34] G. G. Carlo, G. Benenti, G. Casati, and D. L. Shepelyansky, Phys. Rev. Lett. 94, 164101 (2005).

[35] M. Heckel, P. Müller, T. Pöschel, and J. A. C. Gallas, Phys. Rev. E 86, 061310 (2012).

[36] L. Luo, T. J. Tee, and P. L. Chu, J. Opt. Soc. Am. B 15, 972 (1998).

[37] Y. Senlin, Chaos 17, 013106 (2007).

[38] S. Zhang and K. Shen, Chin. Phys. 12, 149 (2003).

[39] A. Endler and J. A. C. Gallas, C. R. Math. (Paris) 342, 681 (2006). 